\title{
How Fading Affects CDMA: An Asymptotic Analysis with Linear Receivers
}

\author{
Ezio Biglieri, Fellow, IEEE, Giuseppe Caire, Member, IEEE, Giorgio Taricco, Member, IEEE, and \\ Emanuele Viterbo, Member, IEEE
}

\begin{abstract}
Using asymptotic analysis, we study the effect of frequency-flat fading on code division multiple access (CDMA) systems with linear receivers and random spreading sequences. Specifically, we let the number of users grow without bound, while the ratio of number of users to spreading sequence length is kept fixed to a value $\alpha$. We treat separately the cases of slow fading (nonergodic channel) and of fast fading (ergodic channel). For the former channel, we derive the outage probability, while for the latter, we compute the channel capacity. In both cases, multiple classes of users with different qualities of service are dealt with. As $\alpha \rightarrow \infty$, the system throughput tends to the same limit of $1.44 \mathrm{bit} / \mathrm{symbol}$ as for the nonfading channel with both single-user matched filter (SUMF) and linear minimum mean-square-error (MMSE) receivers. The outage probability exhibits a floor for al $\alpha$ with the SUMF receiver, while with MMSE receiver the floor is present only for $\alpha \geq 1$. We also address the tradeoffs involved in the allocation of available bandwidth between spreading and coding.
\end{abstract}

Index Terms-CDMA, channel capacity, fading channels, outage probability.

\section{INTRODUCTION}

W E EXAMINE a synchronous code division multiple access (CDMA) single-cell system with error-control coding, operating on a channel affected by frequency-flat fading. The receiver consists of a linear front-end, viz., either a single-user matched filter (SUMF) or a linear minimum-mean-square error (MMSE) filter [14], followed by single-user decoding. The key performance measure here is the signal-to-interference plus noise ratio (SINR) at the output of the linear filter: users' quality of service can be expressed in terms of a target SINR [12]. We treat separately the cases of slow and fast fading, yielding nonergodic and ergodic channels, respectively.

Our study is asymptotic, in the sense that the number of users grows without bound, while the ratio of number of users to spreading-sequence length is kept fixed to a given value $\alpha$. The spreading sequences are random. After the pioneering work of [13] and [15] (see also [9, and references therein]), the asymptotic random-sequences approach emerged as a very powerful tool to characterize in many aspects the behavior of large

Manuscript received November 1, 1999; revised July 11, 2000 and September 30, 2000.

E. Biglieri, G. Taricco and E. Viterbo are with the Dipartimento di Elettronica, Politecnico di Torino, Italy (e-mail: biglieri@polito.it, taricco@polito.it, and viterbo@polito.it).

G. Caire is with the Institut Eurécom, Sophia Antipolis, France (e-mail: giuseppe.caire@eurecom.fr).

Publisher Item Identifier S 0733-8716(01)02953-5.
CDMA systems where the assignment of spreading sequences to the users is pseudorandom (this is the case of the uplink of current CDMA systems like IS-95 or UMTS/IMT2000 [5]). Beyond its theoretical beauty, this method is useful since the performance of actual (finite dimensional) systems converges quickly to the infinite-dimensional asymptotics, which depend only on fundamental system parameters such as the system load (users per chip), the statistics of the received signal-to-noise ratio (SNR) and the constraints on the transmit power, thus making analysis independent on the system fine-tuning characteristics, like the assignment of spreading sequences.

Independent and parallel work on CDMA systems with fading can be found in [6], [17], [16], and [19]. In [6], the random-sequences asymptotic analysis is used to characterize the performance of linear receivers with linear MMSE data-aided channel estimation, both in flat and in multipath channels. The problem of optimal (centralized) power allocation maximizing the system throughput for an optimal joint detector is solved in [17]. Finally, [16] and [19] present system throughput and outage probability analysis for linear and optimal receivers with and without power control, and derives independently the same power control strategy of [17] for the optimal detector. Among the other works in this area, we cite [7, and references therein].

In this paper, we apply the approach of [13] to the case of linear receivers without power control. In the nonergodic case, the SINR cumulative distribution function (cdf) yields immediately the outage probability, i.e., the probability that the actual SINR is below the SINR target. In this case, we show that the outage probability of the SUMF receiver exhibits an error floor for large SNR and all channel loads, while the MMSE receiver does not whenever $\alpha<1$.

In the ergodic case, performance is given in terms of system throughput. Assuming that all users transmit "Gaussian codes," this is also determined by the SINR cdf [2]. In this case, we show that as $\alpha \rightarrow \infty$ the system throughput with the SUMF and MMSE receivers tends to the same limit as for the nonfading channel with the same average SNR. We also showed that there exists a threshold of $E_{b} / N_{0}$ below which the MMSE receiver does not provide any benefit over the SUMF in terms of throughput maximization.

In the case of multiple classes of users with different input power constraints, target SINRs, and outage probability requirements, we find the system capacity region defined as the region of rates at which all user classes can meet their quality of service (QoS) requirements (this region has been defined and studied in [13] in the case of nonfading additive white Gaussian noise 
(AWGN) channel, and should not be confused with the information theoretic capacity region of the multiple access channel [4]).

The paper is organized as follows. After a description of the system model in Section II, we examine the outage probability of slow-fading channels in Section III and the capacity of fast-fading channels in Section IV. The coding-vs.-spreading tradeoff is finally addressed.

\section{SYSTEM MODEL}

We consider the uplink of a single-cell, synchronous direct sequence (DS) CDMA system. Our model involves $K$ users and random spreading sequences of length $L$ chips. As in [13] and [15], we assume a large number of users $(K \rightarrow \infty)$ and $K / L \rightarrow \alpha$ (a constant channel load as the length of the spreading sequences increases to accommodate the $K$ users). Since the system is synchronous, sufficient statistics for (optimal) detection of all users is provided by a chip-matched filter sampled at the chip rate [we assume that the chip waveform satisfies the Nyquist criterion [10] for no interchip interference, so that the sequence of noise samples at the chip matched-filer output is independent identically distributed (i.i.d.)]. The received signal $L$-chip column vector corresponding to one symbol interval is given by

$$
\mathbf{y}=\sum_{k=1}^{K} c_{k} x_{k} \mathbf{s}_{k}+\mathbf{n}
$$

where

n complex circularly-symmetric AWGN vector $\sim \mathcal{N}_{\mathcal{C}}\left(\mathbf{0}, N_{0} \mathbf{I}\right)$

$x_{k} \quad$ complex modulation symbol of user $k$

$\mathbf{s}_{k} \quad$ spreading sequence of user $k$, made of binary antipodal chips $\pm 1 / \sqrt{L}$ generated at random with uniform probability; and

$c_{k} \quad$ frequency-flat complex fading gain, which includes the carrier phase shift of each user and remains constant over the time necessary to transmit a symbol.

We assume that the base station receiver has perfect knowledge of all fading gains (the "channel-state information") and that the demodulation is coherent.

User $k$ is received with an instantaneous SNR $\gamma_{k}=z_{k} \Gamma_{k}$, where $z_{k} \triangleq\left|c_{k}\right|^{2}$ is the fading channel "power gain," $\Gamma_{k}=$ $\mathcal{E}_{k} / N_{0}$ is the transmit SNR and $\mathcal{E}_{k}$ is the user transmit average energy per symbol. We assume that as $K \rightarrow \infty$ the empirical cdf of the received SNRs, defined by

$$
F_{\gamma}^{(K)}(x) \triangleq \frac{1}{K} \sum_{k=1}^{K} \mathbf{1}\left\{\gamma_{k} \leq x\right\}
$$

$(1\{\mathcal{A}\}$ the indicator function of the event $\mathcal{A}$ ) converges almost everywhere to the cumulative distribution function $F_{\gamma}(x)$.

The receiver for user 1 (our reference user) is formed by a linear filter $\mathbf{h}_{1}$ producing the output $y_{1}=\mathbf{h}_{1}^{H} \mathbf{y}$ followed by a single-user decoder operating on the sequence of filter output samples $y_{1}$. We shall consider either the single-user matched filter (SUMF) $\mathbf{h}_{1}=\mathbf{s}_{1}$ and the linear MMSE

$$
\mathbf{h}_{1}=\left[\sum_{k=1}^{K} z_{k} \mathcal{E}_{k} \mathbf{s}_{k} \mathbf{s}_{k}^{H}+N_{0} \mathbf{I}\right]^{-1} \mathbf{s}_{1} .
$$

The SINR at the filter output is defined as

$$
\beta_{1} \triangleq \frac{\gamma_{1}\left|\mathbf{h}_{1}^{H} \mathbf{s}_{1}\right|^{2}}{\left|\mathbf{h}_{1}\right|^{2}+\sum_{k=2}^{K} \gamma_{k}\left|\mathbf{h}_{1}^{H} \mathbf{s}_{k}\right|^{2}} .
$$

Under the above assumptions, we have the following results [13]:

1) The SUMF output SINR converges in probability for large $K$ to

$$
\beta_{1}=\frac{\gamma_{1}}{1+\alpha \int_{0}^{\infty} x d F_{\gamma}(x)} .
$$

2) The MMSE output SINR converges in probability for large $K$ to the (unique) real nonnegative solution of the equation

$$
\beta_{1}=\frac{\gamma_{1}}{1+\alpha \int_{0}^{\infty} \frac{x \gamma_{1}}{\gamma_{1}+x \beta_{1}} d F_{\gamma}(x)} .
$$

\section{A. Distribution of the Output SINR}

From (2), it is immediately apparent that the SINR $\beta_{1}$ is proportional to $\gamma_{1}$. Similarly, since (3) depends only on the ratio $\beta_{1} / \gamma_{1}$, this turns out to be a deterministic quantity. Thus, for both SUMF and MMSE receivers, the SINR has, apart from a scale factor, the same probability distribution as the fading power gain.

\section{B. More than One Class of Users}

We may assume, following [13], that the users are partitioned into $J$ classes, each class $j$ being characterized by a transmit SNR $\Gamma_{j}$. We can think of the $\Gamma_{j}$ s as the transmit SNRs determined by some power-control mechanism, and of the fading $z_{k}$. as some channel attenuation that the power control is not able to compensate, either because it is too fast (as for example with Rayleigh multipath) or because of inaccuracies in the power control loop (as for example with residual shadowing [18]). Each class has $p_{j} K$ users, where $p_{j}$ is the fraction of users belonging to class $j$ (obviously, $\sum_{j=1}^{J} p_{j}=1$ ). Moreover, we assume that the fading gains $z_{k}$ are i.i.d. (the fading statistics is the same for all users) and normalized so that $\int_{0}^{\infty} x d F_{z}(x)=1$. With these assumptions, it is immediate to see that

$$
F_{\gamma}(x)=\sum_{j=1}^{J} p_{j} F_{z}\left(x / \Gamma_{j}\right)
$$

where $F_{z}(x)$ is the fading-gain cdf. Let user 1 belong to class $i$. Because of the uncompensated fading, user-1 SINR is a random 
variable $\beta_{i, 1}$. However, as shown in the previous subsection, the ratio $\xi=\beta_{i, 1} /\left(\Gamma_{i} z_{1}\right)$ is a nonrandom constant independent of $i$. From (2), for the SUMF we obtain

$$
\xi^{(s)}=\frac{1}{1+\alpha \sum_{j=1}^{J} p_{j} \Gamma_{j}}
$$

From (3), for the MMSE, we obtain $\xi=\xi^{(m)}$ as the solution of the equation

$$
\xi=\frac{1}{1+\alpha \sum_{j=1}^{J} p_{j} \Gamma_{j} \int_{0}^{\infty} \frac{x}{1+x \xi \Gamma_{j}} d F_{z}(x)}
$$

This solution is unique, real, and takes values in the interval $[0$, 1]. Moreover, if we rewrite (5) in the form $\xi=g(\xi)$, the iteration $\xi_{n+1}=g\left(\xi_{n}\right)$ converges to the solution for any initial value $\xi_{0}$ [13].

We conclude that any user $k$ belonging to class $i$ has (asymptotically) SINR $\beta_{i, k}=z_{k} \Gamma_{i} \xi$ [where $\xi=\xi^{(s)}$ or $\xi=\xi^{(m)}$ depending on the receiver employed]. Then, the SINR cdf for all users is just given by the fading cdf after a scale change. For users of class $i$, it is given by

$$
F_{i}(x)=\mathbb{P}\left(\beta_{i, k} \leq x\right)=\mathbb{P}\left(z_{k} \leq x /\left(\Gamma_{i} \xi\right)\right)=F_{z}\left(x /\left(\Gamma_{i} \xi\right)\right)
$$

Moreover, the SINRs of different users are (asymptotically as $K \rightarrow \infty)$ statistically independent.

\section{Methodological Preamble}

Here we list a number of points that describe the rationale behind the calculations that follow. We shall analyze a flat, slowfading channel for which the channel gain is constant for the whole duration of a code word, and a flat, fast-fading channel for which the channel gain varies considerably during the transmission of a code word. The information-theoretic subtleties of dealing with fading channels are thoroughly described in [2]. Roughly speaking, we can think of a slow-fading channel as a compound channel, i.e., as a collection of channels each of which is characterized by a fixed set of power gains. An internal channel state process, independent of the input signals and of the noise, selects a particular channel in the compound and keeps this selection for the whole duration of a user codeword. The channel state is known at the receiver and unknown at the transmitters. Each channel in the compound has a well-defined capacity but since the transmitters do not know the channel state realization, they might transmit at a rate above the capacity of the actually selected channel. This event is called information outage, and its probability is the information outage probability. In this setting, the compound channel capacity is not larger than the minimum of the capacities of the channels in the compound. If the infimum of the support of the fading probability distribution is zero (i.e., if there is a nonzero probability that the channel gain is below any assigned positive threshold), the capacity of the slow fading channel is zero. In the case of fast fading, the channel gain experienced during the transmission of a code word varies sufficiently so that all fading realiza- tions occurs with empirical probabilities arbitrarily close to their statistical probabilities. This ergodic behavior of fading makes channel capacity be equal to the channel mutual information $a v$ eraged with respect to the fading statistics (and maximized with respect to the input probability distribution).

Flat, Slow Fading: The outage probability of the reference user is defined by

$$
P_{\text {out }} \triangleq \mathbb{P}(\beta<\bar{\beta}) .
$$

The value of $\bar{\beta}$ may be chosen as follows [3]. For example, if we use a nonideal code with rate $R$ bit/symbol achieving the target performance at a certain $E_{b} / N_{0}$, we set $\bar{\beta}=R E_{b} / N_{0}$. If we consider instead an optimum code which operates at the Shannon limit for a Gaussian channel and we want a rate $R$, then we set

$$
\bar{\beta}=2^{R}-1
$$

Flat, Fast Fading: We assume that all users generate their code book according to a complex circularly-symmetric Gaussian probability density function (pdf); hence, the single-user channel seen at the output of any user's receiving filter is an additive Gaussian noise channel, whose capacity is

$$
C=\mathbb{E}\left[\log _{2}(1+\beta)\right]=\int_{0}^{\infty} \log _{2}(1+x \xi \Gamma) d F_{z}(x) .
$$

System Throughput: The system throughput $\eta$ is defined as the total number of bit/symbol supported by the system. For large systems, it is possible to transmit close to one complex symbol per second per $\mathrm{Hz}$, and hence to express $\eta$ in bit/s/Hz. If all $K$ users transmit at rate $C$, the system throughput is

$$
\eta=\frac{K C}{L}=\alpha C
$$

The value of the channel load that yields maximum throughput is defined as

$$
\alpha_{\mathrm{opt}} \triangleq \arg \max _{\alpha} \eta
$$

\section{Slow-Fading CHANNEL}

Outage probability for users of class $i$ is defined as the probability that the SINR is below some threshold value $\bar{\beta}_{i}$ that depends on the coding scheme of class $i$. We obtain

$$
P_{\text {out }, i}=\mathbb{P}\left(\beta_{i, k} \leq \bar{\beta}_{i}\right)=F_{z}\left(\frac{\bar{\beta}_{i}}{\xi \Gamma_{i}}\right) .
$$

Assuming Gaussian codes and minimum distance decoding at the output of the receiving filter, each user can transmit with arbitrarily small error probability at rate $R_{i, k}=\log _{2}\left(1+\beta_{i, k}\right)$ [8], for sufficiently large code block lengths. Then, in the absence of further specification of class $i$ user codes, it make sense to define the coding rate $R_{i}$ bit/symbol for users in class $i$ and choose the SINR threshold as $\bar{\beta}_{i}=2^{R_{i}}-1$.

\section{A. System Outage Capacity Region}

The system outage capacity is the maximum achievable rate under a given power and outage probability constraints [2]. Here 
we extend the concept to the case of multiple classes and define a system outage capacity region.

Let $\overline{\boldsymbol{\Gamma}}=\left(\bar{\Gamma}_{1}, \ldots, \bar{\Gamma}_{J}\right)$ be a vector of input SNR constraints, $\boldsymbol{\epsilon}=\left(\epsilon_{1}, \ldots, \epsilon_{J}\right)$ a vector of target outage probabilities, and $\mathbf{R}=\left(R_{1}, \ldots, R_{J}\right)$ be a vector of coding rates. We would like to find the set $\mathcal{R} \subseteq \mathbb{R}_{+}^{J}$ of rate vectors $\mathbf{R}$ that can be assigned to the $J$ classes such that, for all $i=1, \ldots, J$

$$
P_{\text {out }, i} \leq \epsilon_{i} \quad \text { and } \quad \Gamma_{i} \leq \bar{\Gamma}_{i}
$$

We refer to the region $\mathcal{R}$ as the system outage capacity region for the input and outage constraints specified by $\overline{\boldsymbol{\Gamma}}$ and $\boldsymbol{\epsilon}$.

SUMF Receiver: Define

$$
\mu_{i} \triangleq \frac{2^{R_{i}}-1}{\sup \left\{x \in \mathbb{R}_{+}: F_{z}(x)=\epsilon_{i}\right\}}
$$

[which reduces to $\mu_{i} \triangleq\left(2^{R_{i}}-1\right) / F_{z}^{-1}\left(\epsilon_{i}\right)$ when $F_{z}(\cdot)$ is strictly monotonic and continuous] and, by using (11) and (4), rewrite the outage constraint as

$$
\frac{\Gamma_{i}}{1+\alpha \sum_{j=1}^{J} p_{j} \Gamma_{j}} \geq \mu_{i} .
$$

The maximum $R_{i}$ is achieved when the above inequalities are satisfied with equality. The solution of the resulting system of equations has the form $\Gamma_{i} / \mu_{i}=\kappa$, where $\kappa$ does not depend on $i$. Solving for $\kappa$ we obtain

$$
\kappa=\frac{1}{1-\alpha \sum_{j=1}^{J} p_{j} \mu_{j}}
$$

provided that

$$
\alpha \sum_{j=1}^{J} p_{j} \mu_{j} \leq 1 .
$$

The resulting transmit SNR assignment (power control) is obtained from $\Gamma_{i}=\kappa \mu_{i}$ in the form

$$
\Gamma_{i}=\frac{\mu_{i}}{1-\alpha \sum_{j=1}^{J} p_{j} \mu_{j}} .
$$

By imposing the input constraint $\Gamma_{i} \leq \bar{\Gamma}_{i}$ for all $i=1, \ldots, J$, we obtain the desired final result

$$
\alpha \sum_{j=1}^{J} p_{j} \mu_{j} \leq \min _{1 \leq i \leq J}\left\{1-\frac{\mu_{i}}{\bar{\Gamma}_{i}}\right\} .
$$

MMSE Receiver: By using (11) and (13), rewrite the outage constraint as

$$
\Gamma_{i} \xi^{(m)} \geq \mu_{i}
$$

Since $\xi^{(m)}$ does not depend on $i$, the solution for the above system (taken with equality) has the form $\Gamma_{i} / \mu_{i}=\kappa$, where $\kappa=1 / \xi^{(m)}$. By substituting in (5) and solving for $\kappa$, we obtain

$$
\kappa=\frac{1}{1-\alpha \sum_{j=1}^{J} p_{j} \mu_{j}^{\prime}}
$$

where

$$
\mu_{j}^{\prime} \triangleq \int_{0}^{\infty} \frac{x \mu_{j}}{1+x \mu_{j}} d F_{z}(x)
$$

provided that

$$
\alpha \sum_{j=1}^{J} p_{j} \mu_{j}^{\prime} \leq 1 .
$$

The resulting transmit SNR assignment (power control) is obtained from $\Gamma_{i}=\kappa \mu_{i}$ in the form

$$
\Gamma_{i}=\frac{\mu_{i}}{1-\alpha \sum_{j=1}^{J} p_{j} \mu_{j}^{\prime}} .
$$

By imposing the input constraint $\Gamma_{i} \leq \bar{\Gamma}_{i}$ for all $i=1, \ldots, J$, we obtain the desired final result

$$
\alpha \sum_{j=1}^{J} p_{j} \mu_{j}^{\prime} \leq \min _{1 \leq i \leq J}\left\{1-\frac{\mu_{i}}{\bar{\Gamma}_{i}}\right\} .
$$

Effective Bandwidth: Equations (15) and (17) are the generalization to the case of slow (nonergodic) fading of the system capacity equations found in [13] in the case of no fading. Interestingly, the outage constraint and the presence of fading yield formally the same constraints for the fractions $\alpha p_{i}$ of class $i$ users per system degree of freedom. In analogy with [13], we define the effective bandwidth $B_{i}$ of class $i$ users as the amount of degrees of freedom consumed in order to support rate $R_{i}$ with outage probability $\epsilon_{i}$. By rewriting (14) in the form

$$
\sum_{j=1}^{J}\left(p_{j} K\right) \mu_{j} \leq L
$$

for the SUMF receiver, we have

$$
B_{i}^{(s)}=\mu_{i} .
$$

Similarly, by rewriting (16) in the form

$$
\sum_{j=1}^{J}\left(p_{j} K\right) \mu_{j}^{\prime} \leq L
$$

for the MMSE receiver, we have

$$
B_{i}^{(m)}=\mu_{j}^{\prime} \text {. }
$$

Obviously, $B_{i}^{(s)} \geq B_{i}^{(m)}$ and $B_{i}^{(m)} \leq 1$. For very high quality of service (large rates and/or small outage probabilities) $\mu_{i}$ becomes large. We observe that, with SUMF receiver, class- $i$ users may require an unbounded number of degrees of freedom, while 
with MMSE receiver they will require at most one degree of freedom, as in the absence of fading [13].

\section{B. Outage Probability Floor and Near-Far Resistance}

Consider the case of a single class, and neglect for simplicity the class index. The outage probability is given by $P_{\text {out }}=$ $F_{z}(\bar{\beta} /(\xi \Gamma))$. As $\Gamma \rightarrow \infty$, for the SUMF receiver, we have

$$
\lim _{\Gamma \rightarrow \infty} \xi^{(s)} \Gamma=1 / \alpha
$$

Therefore, the outage probability has a floor at $F_{z}(\alpha \bar{\beta})$ for all $\alpha>0$. This is a consequence of the fact that CDMA with SUMF reception is interference-limited, and because of fading, there is always a nonzero probability that some interferer is so strong that drives the SINR below the target threshold.

With an MMSE receiver, if we let $\Gamma \rightarrow \infty$ in (5), we obtain the equation

$$
\frac{\xi^{(m)}}{\xi^{(m)}+\alpha}=\xi^{(m)} .
$$

This has a positive solution for $\xi^{(m)}$ if and only if $\alpha<1$ [recall that $0 \leq \xi^{(m)} \leq 1$ ], otherwise $\xi^{(m)}=0$ is the only solution. We conclude that, if $\alpha<1$ then $\lim _{\Gamma \rightarrow \infty} P_{\text {out }}=0$ (no outage probability floor), otherwise there is an error floor. This is a consequence of the fact that the MMSE receiver is near-far resistant (i.e., not interference-limited) if the user spreading sequences are linearly independent, and that with sufficiently long random sequences linear independence is achieved with arbitrarily large probability if $\alpha<1$ and with arbitrarily small probability if $\alpha>1$ [14].

Example 1: Assume for the fading gains a log-normal distribution with log-standard deviation $\sigma \mathrm{dB}$ (the "shadowing factor") and mean value $\mathbb{E}[z]=1$. Letting $\xi^{(s)} \triangleq 1 /\left(\Gamma^{-1}+\alpha\right)$, for the SUMF, we get

$$
P_{\text {out }}^{\mathrm{s}}=1-Q\left[\sigma^{-1}\left(\mu_{z}+10 \log _{10}\left[\left(2^{R}-1\right) / \xi^{(s)}\right]\right)\right]
$$

where $Q(x) \triangleq \mathbb{P}(\mathcal{N}(0,1)>x)$. For the MMSE, we obtain the equation

$$
z_{1} / \beta=\Gamma^{-1}+\alpha \mathbb{E}_{z}\left[\frac{z}{z_{1} / \beta+z}\right]\left(z_{1} / \beta\right)
$$

which can be solved iteratively through the recursion

$$
\begin{aligned}
\xi_{n} & =\Gamma^{-1}+\alpha \mathbb{E}\left[\frac{z}{\xi_{n-1}+z}\right] \xi_{n-1} \\
& =\Gamma^{-1}+\alpha \mathbb{E}_{X}\left[\frac{10^{\sigma X / 10}}{\xi_{n-1} 10^{\mu_{z} / 10}+10^{\sigma X / 10}}\right] \xi_{n-1} \\
& =\Gamma^{-1}+\frac{\alpha}{\sqrt{2 \pi}} \xi_{n-1} \int_{-\infty}^{\infty} \frac{e^{-x^{2} / 2}}{\xi_{n-1} 10^{\left(\mu_{z}-\sigma x\right) / 10}+1} d x
\end{aligned}
$$

where $X \sim \mathcal{N}(0,1)$, with the initial value $\xi_{0}=1$. This iteration converges to $\lim _{n \rightarrow \infty} \xi_{n}=z_{1} / \beta$. The computation of the outage probability reduces to determining the cumulative distribution function of a log-normal variates:

$$
P_{\mathrm{out}}^{\mathrm{s}}=1-Q\left[\sigma^{-1}\left(\mu_{z}+10 \log _{10}\left[\left(2^{R}-1\right) / \xi^{(m)}\right)\right] .\right.
$$

With the SUMF receiver, since $\lim _{\Gamma \rightarrow \infty} \xi^{(s)}=\alpha^{-1}$, the outage probability floor is

$$
1-Q\left[\sigma^{-1}\left(\mu_{z}+10 \log _{10}\left[\left(2^{R}-1\right) \alpha\right]\right)\right]
$$

for any value of $\alpha$.

The outage probability, as well as its floor, is illustrated in Fig. 1, obtained by plotting $P_{\text {out }}$ versus $E_{b} / N_{0}$ with the MMSE and SUMF receivers, $\alpha=0.2,0.5,0.8,1,1.2,1.5, \bar{\beta}=2^{R}-$ 1 with rates $R=1$ and 2 bit/symbol, and log-normal fading with shadowing factor [11] $\sigma=2$ and $8 \mathrm{~dB}$. The outage probability degrades, as expected, by increasing either $R, \alpha$, or $\sigma$, which represent the user rate, system load, and shadowing level, respectively.

Fig. 2 shows the outage capacity region with SUMF and MMSE receiver in a system with two user groups: the first one has $90 \%$ of the users transmitting with an outage probability 0.1 and a SNR of $\bar{\Gamma}_{1}=10 \mathrm{~dB}$; the second one has $10 \%$ of the users transmitting with an outage probability 0.01 and a SNR of $\bar{\Gamma}_{2}=13 \mathrm{~dB}$. The channel statistics are log-normal with $\log$-standard deviation $\sigma=2 \mathrm{~dB}$, and $\alpha=0.2,0.5,1$, and 2 .

\section{ERGODIC FADING}

Consider again $J$ classes of users. From (9), the rate at which a user in class $i$ can communicate reliably in an ergodic-fading regime is given by

$$
R_{i}=\int_{0}^{\infty} \log _{2}\left(1+x \xi \Gamma_{i}\right) d F_{z}(x)
$$

where $\xi=\xi^{(s)}$ or $\xi=\xi^{(m)}$ depending on the linear receiver employed. We want to determine the set of rates $\mathbf{R}=\left(R_{1}, \ldots, R_{J}\right)$ achievable by the system with input constraint $\boldsymbol{\Gamma} \leq \overline{\boldsymbol{\Gamma}}$, load $\alpha$, and fractions $p_{1}, \ldots, p_{J}$ of users belonging to classes $1, \ldots, J$.

Let $f(y) \triangleq \int_{0}^{\infty} \log _{2}(1+x y) d F_{z}(x)$. This function is monotonically increasing for $y \geq 0$. Next, for all $i=1, \ldots, J$, define

$$
\nu_{i} \triangleq f^{-1}\left(R_{i}\right)
$$

The solution of the rate equations (20) with respect to the $\Gamma_{i} \mathrm{~s}$ has the form

$$
\xi \Gamma_{i}=\nu_{i}
$$

i.e., $\Gamma_{i} / \nu_{i}=1 / \xi$, a constant independent of $i$.

We can now see that the problem we are dealing with here is formally identical to that solved in previous section and leading to the outage capacity region: thus, power control, capacity, and effective bandwidth formulas in the ergodic case can be obtained simply by replacing $\nu_{j}$ for $\mu_{j}$ in the results for the outage capacity. Explicitly, for the SUMF receiver, we have

$$
\xi=1-\alpha \sum_{j=1}^{J} p_{j} \nu_{j}
$$



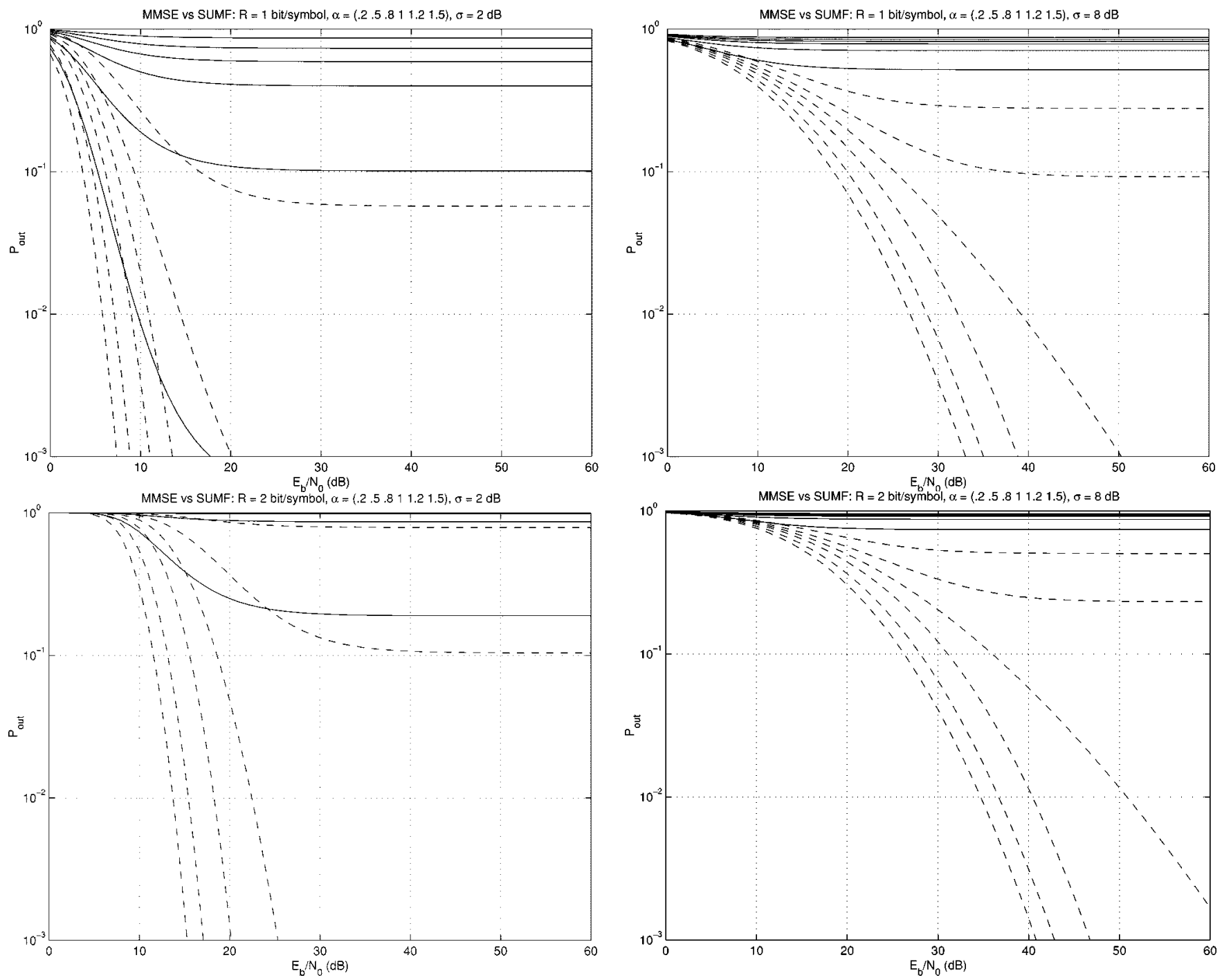

Fig. 1. Outage probability with MMSE (dashed) and SUMF (solid) for rates $R=1$ and 2 bit/symbol, $\sigma=2$ and $8 \mathrm{~dB}$, and $\alpha=0.2,0.5,0.8,1,1.2,1.5$.

provided that

$$
\alpha \sum_{j=1}^{J} p_{j} \nu_{j} \leq 1
$$

The power-control equation is

$$
\Gamma_{i}=\frac{\nu_{i}}{1-\alpha \sum_{j=1}^{J} p_{j} \nu_{j}}
$$

and, by imposing the input constraint $\Gamma_{i} \leq \bar{\Gamma}_{i}$ for all $i=$ $1, \ldots, J$, we obtain the capacity inequality

$$
\alpha \sum_{j=1}^{J} p_{j} \nu_{j} \leq \min _{1 \leq i \leq J}\left\{1-\frac{\nu_{i}}{\bar{\Gamma}_{i}}\right\} .
$$

For the MMSE receiver, we obtain

$$
\xi=1-\alpha \sum_{j=1}^{J} p_{j} \nu_{j}^{\prime}
$$

where

$$
\nu_{j}^{\prime} \triangleq \int_{0}^{\infty} \frac{x \nu_{j}}{1+x \nu_{j}} d F_{z}(x)
$$

provided that

$$
\alpha \sum_{j=1}^{J} p_{j} \nu_{j}^{\prime} \leq 1
$$

The resulting power-control equation is

$$
\Gamma_{i}=\frac{\nu_{i}}{1-\alpha \sum_{j=1}^{J} p_{j} \nu_{j}^{\prime}}
$$

By imposing the input constraint $\Gamma_{i} \leq \bar{\Gamma}_{i}$ for all $i=1, \ldots, J$, we obtain the capacity inequality

$$
\alpha \sum_{j=1}^{J} p_{j} \nu_{j}^{\prime} \leq \min _{1 \leq i \leq J}\left\{1-\frac{\nu_{i}}{\bar{\Gamma}_{i}}\right\} .
$$




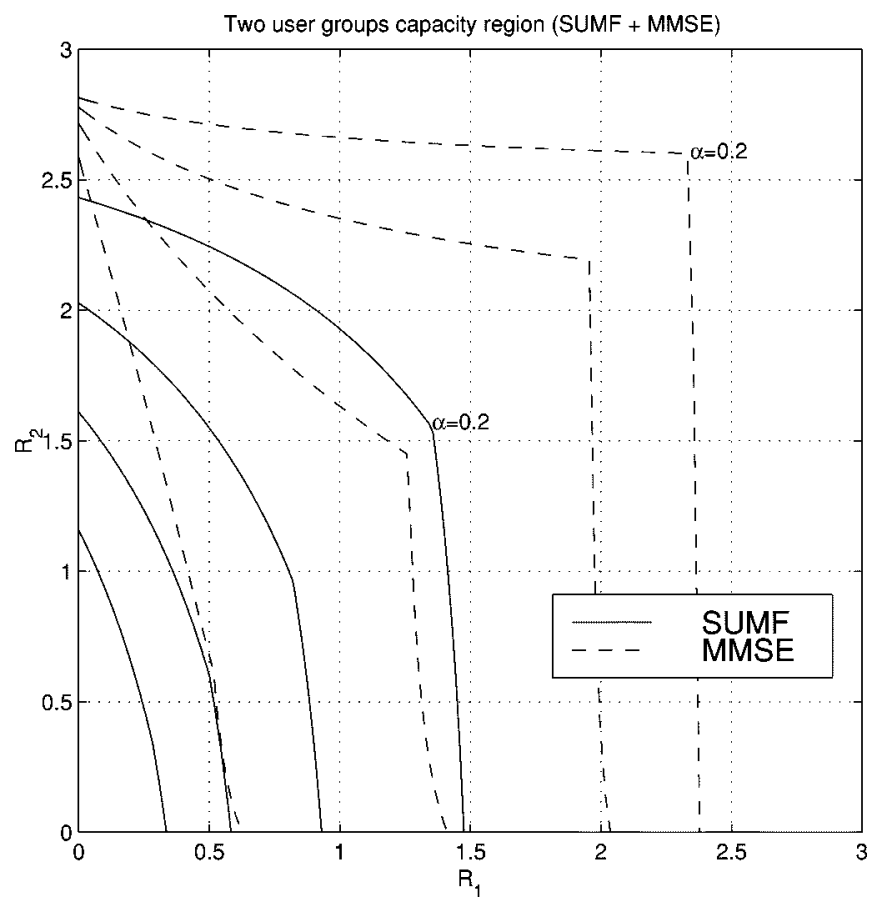

Fig. 2. System outage capacity regions with two user groups. The first one has $90 \%$ of the users transmitting with an outage probability $P_{\text {out }, 1}=0.1$ and a SNR $\bar{\Gamma}_{1}=10 \mathrm{~dB}$; the second one has $10 \%$ of the users transmitting with an outage probability $P_{\text {out, } 2}=0.01$ and a signal-to-noise ratio $\bar{\Gamma}_{2}=13 \mathrm{~dB}$. The channel statistics are log-normal with log-standard deviation $\sigma=2 \mathrm{~dB}$, and $\alpha=0.2,0.5,1$ and 2 .

The effective bandwidth is given by

$$
B_{i}^{(s)}=\nu_{i}
$$

for the SUMF and by

$$
B_{i}^{(m)}=\nu_{j}^{\prime}
$$

for the MMSE.

Example 2: Assume Rayleigh fading, i.e., $F_{z}(x)=(1-$ $\exp (-x)) u(x)$. From (9), we have

$$
C=e^{1 /(\xi \Gamma)} \operatorname{Ei}(1,1 /(\xi \Gamma)) \log _{2} e
$$

where

$$
\operatorname{Ei}(1, x) \triangleq \int_{1}^{\infty} \frac{e^{-x t}}{t} d t
$$

and $\xi=\xi^{(s)}$ or $\xi=\xi^{(m)}$, depending on the receiver used.

Fig. 3 shows the capacity curves for SUMF and MMSE as a function of $E_{b} / N_{0}$ for different values of $\alpha$ with Rayleigh fading. With the SUMF, the capacity is bounded for all $\alpha$, while with the MMSE it is bounded only for $\alpha \geq 1$ (interference-limited condition). Fig. 4 shows the system capacity regions with SUMF and MMSE receiver in a system with two user groups: the first one has $90 \%$ of the users transmitting with a SNR 10 $\mathrm{dB}$, and the second has the remaining users with a SNR $13 \mathrm{~dB}$.

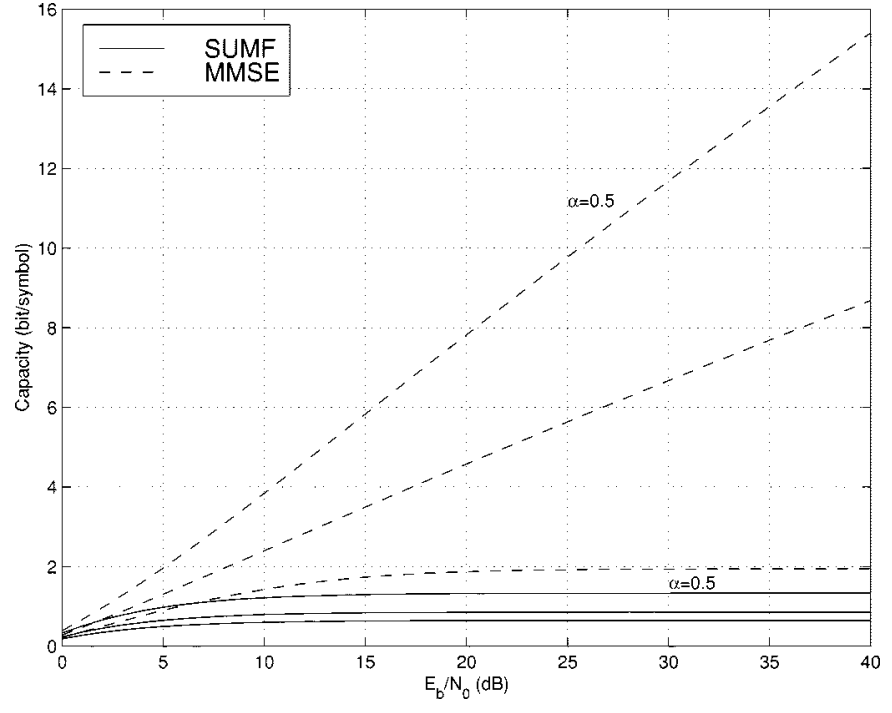

Fig. 3. Capacity of MMSE (dashed) and SUMF (solid) (bit/symbol), $\alpha=$ $0.5,1,1.5$.

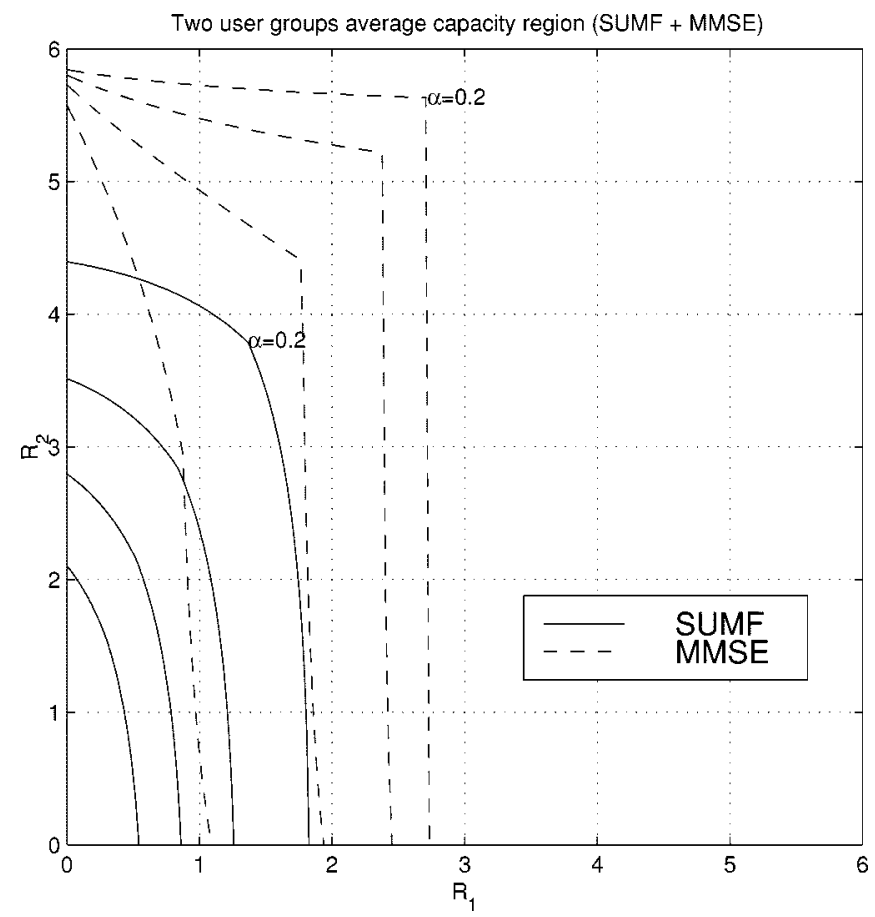

Fig. 4. System capacity regions with two user groups. The first one has $90 \%$ of the users transmitting with a SNR $10 \mathrm{~dB}$; the second one has $10 \%$ of the users transmitting with a SNR $13 \mathrm{~dB}$. The channel statistics are Rayleigh, and $\alpha=0.2,0.5,1$, and 2 .

\section{A. System Throughput}

Let $\tilde{\xi}=\beta_{1} / z_{1}$ and $\Gamma=R E_{b} / N_{0}$. Then, (2) and (3) can be rewritten in the form

$$
\tilde{\xi}=\frac{R(\tilde{\xi}) \frac{E_{b}}{N_{0}}}{1+\eta \frac{E_{b}}{N_{0}} \zeta(\tilde{\xi})}
$$


where $R(\tilde{\xi})=E\left[\log _{2}(1+\tilde{\xi} z)\right]$ and where

$$
\zeta(\tilde{\xi})= \begin{cases}E[z] & \text { SUMF } \\ E\left[\frac{z}{1+z \tilde{\xi}}\right] & \text { MMSE. }\end{cases}
$$

By rearranging the terms in (24), we can express directly the throughput $\eta$ as a function of $\tilde{\xi}$, namely

$$
\eta=\frac{1}{\zeta(\tilde{\xi})}\left[\frac{R(\tilde{\xi})}{\tilde{\xi}}-\frac{1}{E_{b} / N_{0}}\right]_{+} .
$$

Assuming $E[z]=1$, we have that

$$
\lim _{\tilde{\xi} \rightarrow 0} \eta=\left[\log _{2} e-\frac{1}{E_{b} / N_{0}}\right]_{+}
$$

which is positive for $E_{b} / N_{0}>\log 2=-1.59 \mathrm{~dB}$. Moreover, from (24) and from the fact that $\eta=\alpha R(\tilde{\xi})$, it is immediate to see that $\tilde{\xi}$ vanishes as $\alpha \rightarrow \infty$. Then, for both the SUMF and the MMSE receivers, we have that (26) is also the limit of $\eta$ for large channel load $\alpha$ (this limit is the same as for a nonfading channel [15]).

The function $\eta(\tilde{\xi})$ defined by (25) is nonincreasing for all $E_{b} / N_{0}$ for the SUMF. This implies that the maximum $\eta$ with SUMF reception is obtained for $\alpha \rightarrow \infty$ and vanishing per-user rate $R(\tilde{\xi}) \rightarrow 0$. With MMSE reception, $\eta(\tilde{\xi})$ is nonincreasing for $E_{b} / N_{0} \leq\left(E_{b} / N_{0}\right)_{\mathrm{th}}$, while it has a maximum for positive $\tilde{\xi}$ for $E_{b} / N_{0}>\left(E_{b} / N_{0}\right)_{\text {th }}$ where $\left(E_{b} / N_{0}\right)_{\text {th }}$ is a threshold value (the same behavior is noticed in a nonfading channel [15], [3]). Remarkably, for $E_{b} / N_{0} \leq\left(E_{b} / N_{0}\right)_{\text {th }}$, the system throughput is maximized by $\alpha \rightarrow \infty$. Therefore, there is no point in using a MMSE detector since the same maximum throughput is achieved by the SUMF detector. We can calculate explicitly the value of $\left(E_{b} / N_{0}\right)_{\text {th }}$ by solving the inequality

$$
\left.\frac{\partial \eta(\tilde{\xi})}{\partial \tilde{\xi}}\right|_{\tilde{\xi}=0} \geq 0 .
$$

For all fading distributions with $E[z]=1$, we obtain $\left(E_{b} / N_{0}\right)_{\mathrm{th}}=2 \log 2=1.41 \mathrm{~dB}$ (the same value as for a nonfading channel [3]). Fig. 5 shows the qualitative behavior of $\eta$ as a function of $\tilde{\xi}$ for $E_{b} / N_{0}$ below and above the threshold, for SUMF and MMSE. Figs. 6 and 7 show both $C$ and $\eta$ vs. $\alpha$ at $E_{b} / N_{0}=1 \mathrm{~dB}$ and $6 \mathrm{~dB}$, respectively, for Rayleigh fading and a channel with no fading. The presence of a maximum throughput for finite $\alpha$ when $E_{b} / N_{0}>\left(E_{b} / N_{0}\right)_{\text {th }}$ for the MMSE receiver is clearly visible.

Fig. 8 shows the system throughput optimized with respect to $\alpha$ as a function of $E_{b} / N_{0}$, for the SUMF and MMSE receivers, for Rayleigh fading and no fading (analogous results are shown in [16], [19] also for other types of receivers with different power control strategies).

Finally, Fig. 9 shows the behavior of $\alpha_{\mathrm{opt}}$ as a function of $E_{b} / N_{0}$ for the MMSE receiver with and without Rayleigh fading. As $E_{b} / N_{0} \rightarrow\left(E_{b} / N_{0}\right)_{\text {th }}$ from the right, $\alpha_{\text {opt }}$ diverges. In the range $\log 2 \leq E_{b} / N_{0} \leq 2 \log 2, \alpha_{\text {opt }} \rightarrow \infty$ and MMSE reception is useless for the sake of maximizing the throughput. Notice that $\alpha_{\mathrm{opt}}$ exhibits a minimum (this occurs

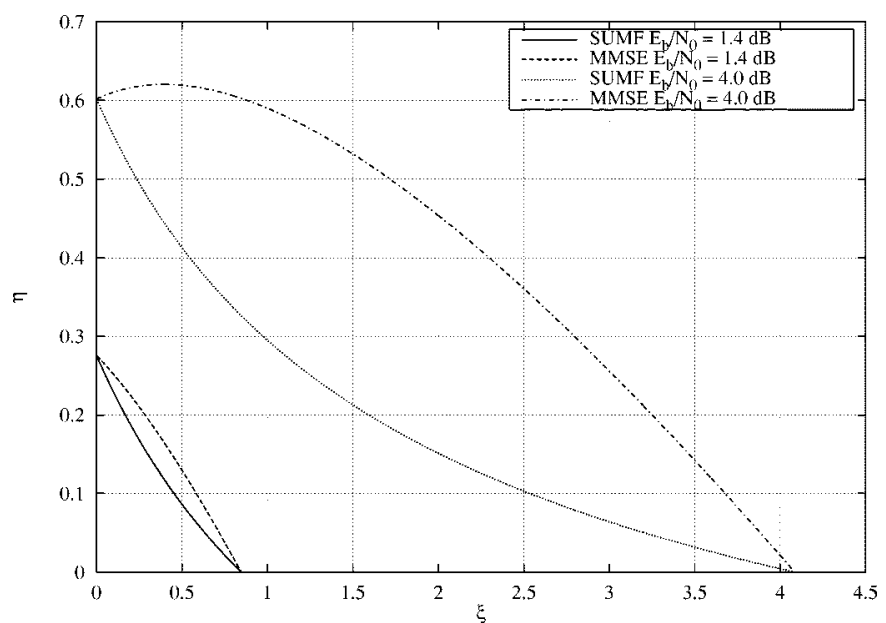

Fig. 5. Qualitative behavior of $\eta$ as a function of $\tilde{\xi}$ for $E_{b} / N_{0}$ below and above the threshold, for SUMF and MMSE.

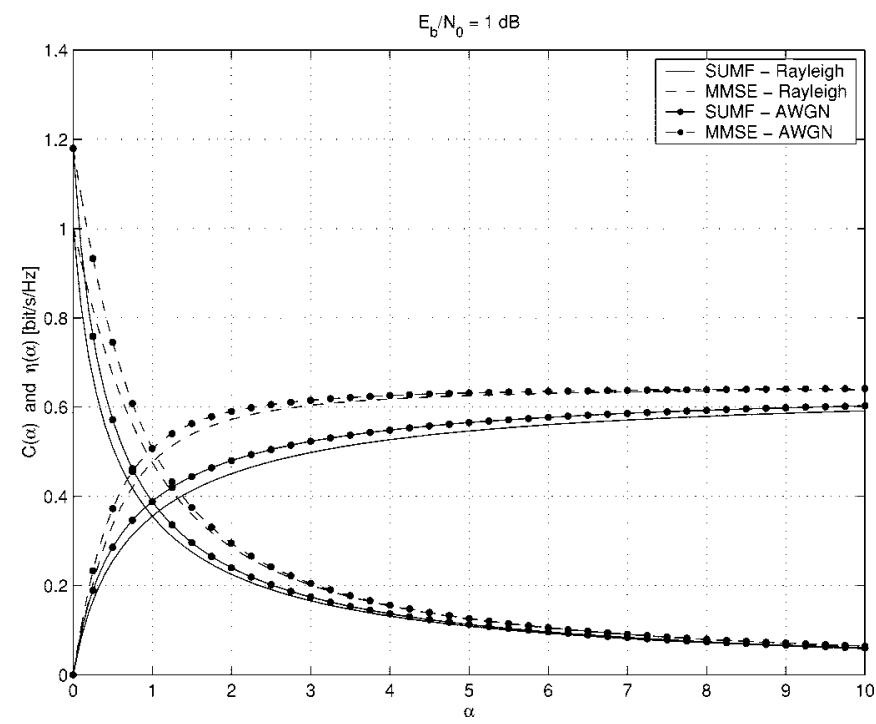

Fig. 6. Plot of $C(\alpha)$-monotonically decreasing curves—and $\eta(\alpha)$ vs. $\alpha$ for the MMSE (dashed) and SUMF (solid) receiver for $E_{b} / N_{0}=1 \mathrm{~dB}$.

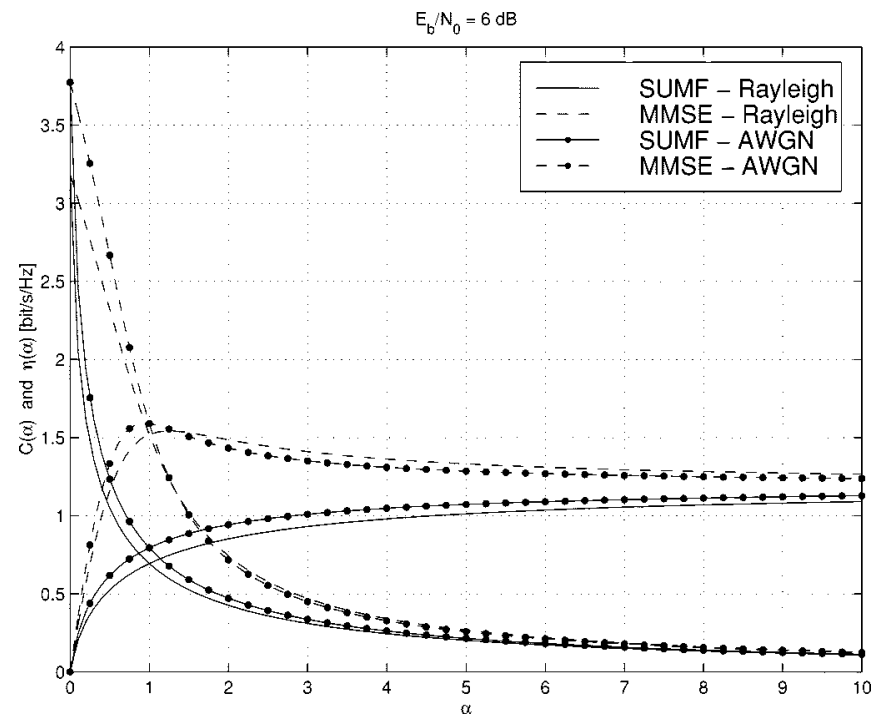

Fig. 7. Plot of $C(\alpha)$-monotonically decreasing curves—and $\eta(\alpha)$ vs. $\alpha$ for the MMSE (dashed) and SUMF (solid) receiver for $E_{b} / N_{0}=6 \mathrm{~dB}$. 


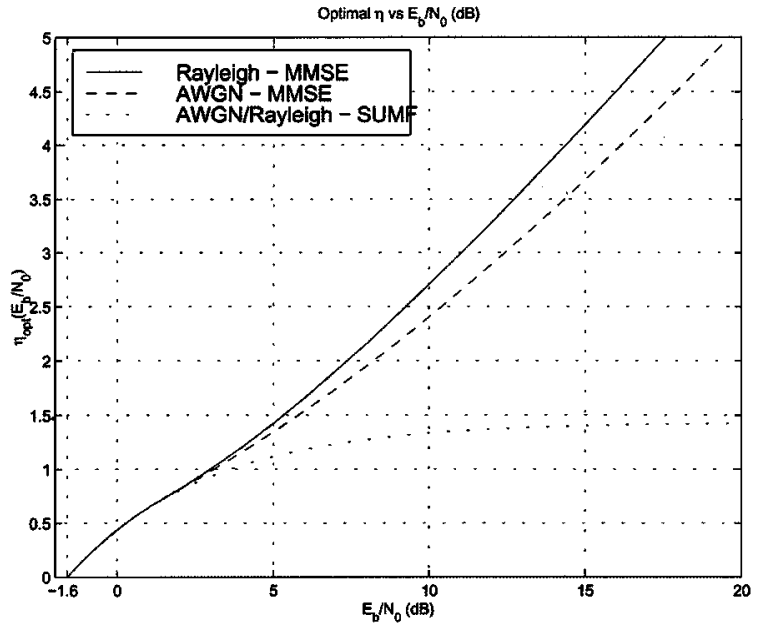

Fig. 8. Optimal system throughput $\eta_{\text {opt }}$ versus $E_{b} / N_{0}$ for SUMF and MMSE receivers: AWGN and Rayleigh fading channel.

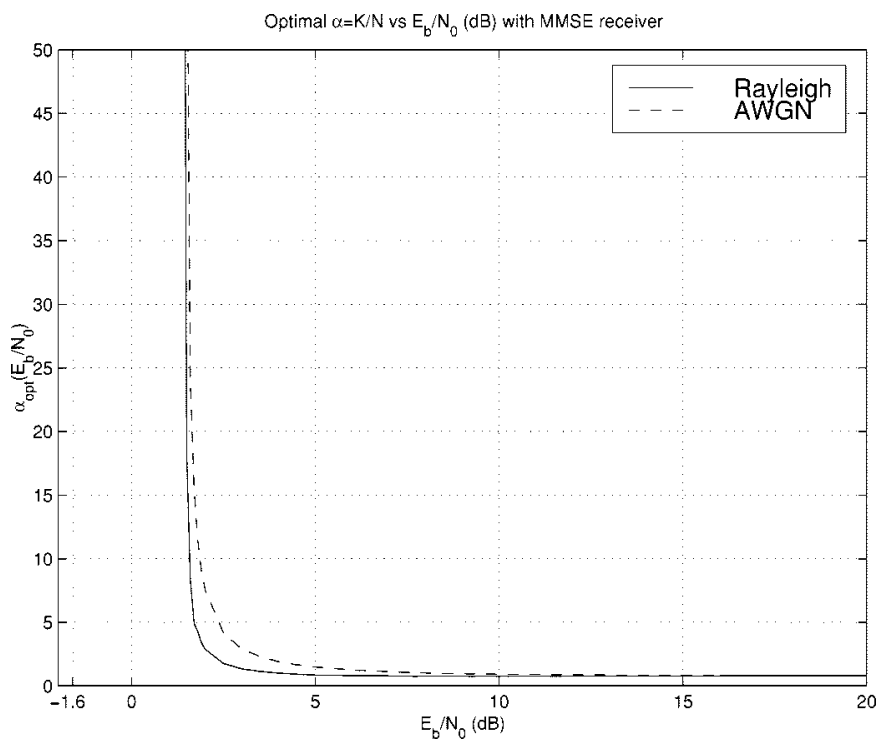

Fig. 9. $\alpha_{\text {opt }}$ versus $E_{b} / N_{0}$ for the MMSE receiver: AWGN and Rayleigh fading channel.

at $E_{b} / N_{0} \approx 10$ and $\approx 18 \mathrm{~dB}$ for no fading and Rayleigh fading, respectively). This behavior can be explained by noting that for low $E_{b} / N_{0}$, the MMSE receiver approaches the SUMF [noise dominates multiple access interference (MAI) in this case], and the system throughput is maximum when $\alpha$ is large. For high $E_{b} / N_{0}$, the MMSE receiver approaches the decorrelating detector [14] (this is the optimal linear receiver in the absence of noise), and system throughput is maximum for $\alpha \rightarrow 1$. Moreover, $\alpha$ must tend to one from below, since for large $\Gamma$ we get $\alpha \simeq 1-\xi^{(m)}$ with $\xi^{(m)} \leq 1$. Then, by continuity, $\alpha$ must have a minimum for some $E_{b} / N_{0}>2 \log 2$.

As far as the effect of fading on the system throughput is concerned, Rayleigh fading always decreases throughput with the SUMF, while for large $\alpha$ and MMSE detection it provides a modest throughput increase. This can be interpreted as a sort of implicit "load control" operated by fading (see [16], [19]): the fraction of relevant interferers per chip is actually smaller than $\alpha$ because some users experience deep fading. The dimensional crowding problem of the linear MMSE receiver is alleviated by fading, and for large $\alpha$ the benefit of this effect is larger than the degradation due to the fading of the useful signal component.

\section{B. Spreading-Coding Tradeoff}

We use the above analysis to dimension a nonasymptotic CDMA system with total bandwidth $W$, user information bit-rate $R_{b}$ and transmit power $P$. The energy per bit is given by $E_{b}=P / R_{b}$ and the bandwidth expansion is given by $W / R_{b}$. Since $P, W$ and $R_{b}$ are system constraints and we assume $N_{0}$ given, both $E_{b} / N_{0}$ and $W / R_{b}$ are fixed. The bandwidth expansion should be apportioned between spreading and coding, so that $L / R=W / R_{b}$, where $R$ is the user coding rate, expressed in information bits per symbol, and $L$ is the spreading factor, expressed in dimensions per symbol. By optimal spreading-coding trade-off, we mean to dimension the system so that $\eta$ is maximum, i.e., select $\alpha=\alpha_{\text {opt }}$ and $R=C\left(\alpha_{\text {opt }}\right)$. As a consequence, the optimized spreading gain is obtained as $L=\left(W / R_{b}\right) C\left(\alpha_{\text {opt }}\right)$. For a system based on SUMF, the system throughput is maximized for $\alpha \rightarrow \infty$. This implies a very large number of users, each of which transmitting at a low coding rate. In this case, as it is well-known, the whole bandwidth expansion should be devoted to (low-rate) coding, while devoting a minimum amount of spreading to acquisition and synchronization [18]. On the contrary, for a system equipped with an MMSE receiver, we observe that for $E_{b} / N_{0}>\left(E_{b} / N_{0}\right)_{\text {th }}$ there is a finite $\alpha_{\text {opt }}$, otherwise $\alpha_{\text {opt }}$ is infinite and SUMF is good enough.

Example 3: Consider a system with parameters $W=4$ $\mathrm{MHz}, R_{b}=16 \mathrm{~kb} / \mathrm{s}$ and $E_{b} / N_{0}=6 \mathrm{~dB}$ (these figures are inspired by UMTS [5]). From Fig. 7, we see that with MMSE $\alpha_{\text {opt }} \approx 1.3$ and $C\left(\alpha_{\text {opt }}\right) \approx 1.2$. This yields $L=300$ and $K=390$. A coding rate of $1.2 \mathrm{bit} / \mathrm{symbol}$ can be approximated, for example, by binary turbo coding of rate 4/7 concatenated with QPSK, or binary coding of rate $3 / 7$ concatenated with 8PSK, where efficient implementations for binary coding rates 4/7 and 3/7 can be obtained by suitably puncturing mother codes of rate $1 / n$ [1]. Practical system values with conventional techniques are $L=256$ and $K \leq 100$ [5]. Therefore, numbers provided by asymptotic analysis appear quite realistic and, in passing, show the potential benefit of linear interference rejection techniques and powerful channel coding, at least in the case of an isolated cell.

\section{CONCLUSION}

In this paper, we examined a CDMA system operating on a channel affected by frequency-flat fading. The receiver consists of either a SUMF or an MMSE filter. The cases of slow and fast fading have been considered separately, yielding nonergodic and ergodic channels, respectively. In the nonergodic case, we studied the outage probability and the system outage capacity. In the ergodic case, performance was expressed in terms of system throughput and system capacity. Among our findings, we showed that, in a slow-fading regime, the outage probability of the SUMF receiver exhibits an error floor for large SNR and all channel loads, while the MMSE receiver does not whenever $\alpha<1$. Also, we showed that, in a fast-fading regime as $\alpha \rightarrow \infty$, the system throughput with SUMF and MMSE tends 
to the same limit as for the nonfading channel with the same average SNR. Moreover, we showed that there exists a threshold of $E_{b} / N_{0}$ below which the MMSE receiver does not provide any benefit over the SUMF in terms of throughput maximization. Finally, we have addressed the tradeoffs involved in the allocation of available bandwidth between spreading and coding, and we showed that the asymptotic analysis based on random spreading sequences gives actually meaningful and easy-to-compute results and may serve as a tool to dimension practical finite-size systems.

\section{ACKNOWLEDGMENT}

The authors are grateful to the anonymous reviewers for their useful comments and, in particular, for calling their attention on reference [7].

\section{REFERENCES}

[1] C. Berrou, A. Glavieux, and P. Thitimajshima, "Near Shannon limit error-correcting coding and decoding: Turbo-codes," in IEEE Int. Conf. Commun., Geneva, Switzerland, May 1993, pp. 1064-1070.

[2] E. Biglieri, J. Proakis, and S. Shamai (Shitz), "Fading channels: Information-theoretic and communications aspects," IEEE Trans. Inform. Theory, vol. 44, pp. 2619-2692, Oct. 1998.

[3] G. Caire, G. Taricco, and E. Biglieri, "CDMA system design through asymptotic analysis,", 1999, submitted for publication.

[4] T. Cover and J. Thomas, Elements of Information Theory. New York: Wiley, 1991.

[5] E. Dahlman, B. Gudmundson, M. Nilsson, and J. Sköld, "UMTS/IMT-2000 based on wideband CDMA," IEEE Commun. Mag., vol. 36, pp. 70-80, Sept. 1998.

[6] J. Evans and D. Tse, "Large system performance of linear multiuser receivers in multipath fading channels," IEEE Trans. Inform. Theory, 2000.

[7] A. Lampe and J. B. Huber, "On the limits of coded transmission over fading channels with CDMA," in 3rd ITG Conf. Source and Channel Coding, Munich, Germany, January 17-19, 2000, pp. 313-318.

[8] A. Lapidoth, "Nearest-neighbor decoding for additive non-Gaussian noise channels," IEEE Trans. Inform. Theory, vol. 42, pp. 1520-1529, Sept. 1996.

[9] R. Müller, "Power and bandwidth efficiency of multiuser systems with random spreading," Ph.D. dissertation, Universität Erlangen-Nürnberg, 1999.

[10] J. Proakis, Digital Communications, 3rd ed. New York: McGraw-Hill, 1995.

[11] T. Rappaport, Wireless Communications. Englewood Cliffs, NJ: Prentice-Hall, 1996.

[12] D. Tse and S. V. Hanly, "Network capacity, power control, and effective bandwidth," in Wireless Communications: Signal Processing Perspectives, ser. Prentice-Hall, H. Vincent Poor and G. W. Wornell, Eds. Englewood Cliffs, NJ, 1998

[13] D. Tse and S. V. Hanly, "Linear multiuser receivers: Effective interference, effective bandwidth and capacity," IEEE Trans. Inform. Theory, vol. 45, pp. 641-657, Mar. 1999.

[14] S. Verdú, Multiuser Detection. New York: Cambridge Univ. Press, 1998.

[15] S. Verdú and S. Shamai (Shitz), "Spectral efficiency of CDMA with random spreading," IEEE Trans. Inform. Theory, vol. 45, pp. 622-640, Mar. 1999.

[16] S. Verdú and S. Shamai (Shitz), "The effect of frequency-flat fading on the spectral efficiency of CDMA," IEEE Trans. Inform. Theory, Nov. 1999, submitted for publication.

[17] P. Viswanath, D. Tse, and V. Anantharam, "Asymptotically optimal waterfilling in vector multiple access channels," IEEE Trans. Inform. Theory, Nov. 1999, submitted for publication.

[18] A. J. Viterbi, CDMA-Principles of Spread-Spectrum Communications. Reading, MA: Addison-Wesley, 1995.

[19] S. Verdú and S. Shamai (Shitz), "Capacity of CDMA fading channels," in IEEE Inform. Theory Workshop, Metsovo, Greece, June 27-July 1, 1999.

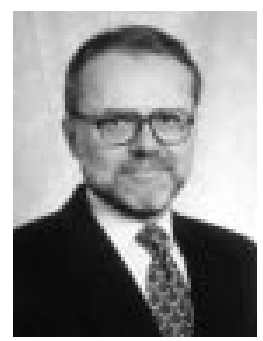

Ezio Biglieri (M'73-SM'82-F'89) was born in Aosta, Italy. He received the Dr.Engr. degree in electrical engineering from Politecnico di Torino, Italy in 1967.

From 1968 to 1975 , he was with the Instituto di Elettronica a Telecomunicazioni, Politecnico di Torino, first as a Research Engineer, then as an Associate Professor (jointly with Instituto Matematico). In 1975, he was made a Professor of Electrical Engineering with the University of Napoli, Italy. In 1977, he returned to Politecnico di Torino as a Professor with the Department of Electrical Engineering. From 1987 to 1989, he was a Professor of Electrical Engineering with the University of California, Los Angeles. Since 1990, he has been again a Professor with Politecnico di Torino.

He has held visiting positions with the Department of System Science, UCLA, the Mathematical Research Center, Bell Laboratories, Murray Hill, NJ, the Bell Laboratories, Holmdel, NJ, the Department of Electrical Engineering, UCLA, the Telecommunication Department of The Ecole Nationale Supérieure des Télécommunications, Paris, France, the University of Sydney, Australia, the Yokohama National University, Japan, and the Electrical Engineering Department of Princeton University.

In 1988, 1992, and 1996, Dr. Biglieri was elected to the Board of Governors of the IEEE Information Theory society, in which he served as its President in 1999. From 1988 to 1991, he was an Editor of the IEEE TRANSACTIONS ON COMMUNICATIONS, and from 1991 to 1994, he was an Associate Editor of the IEEE TRANSACTIONS ON INFORMATION THEORY. Since 1997, he has been an Editor of the IEEE Communications Letters and the Editor in Chief of the European Transactions on Telecommunications, and since 1998 a Division Editor of the Journal on Communications and Networks. He has edited three books and coauthored five, among which the recent Principles of Digital Transmission with Wireless Applications (New York: Kluwerslash Plenum, 1999). In 2000, he received the IEEE Third-Millennium Medal and the IEEE Donald G. Fink Prize Paper Award.

Giuseppe Caire (S'91-M'94) was born in Torino, Italy, on May 21, 1965. He received the B.Sc. degree in electrical engineering from Politecnico di Torino, Italy, in 1990, the M.Sc. degree in electrical engineering from Princeton University, Princeton, NJ in 1992, and the Ph.D. degree from Politecnico di Torino in 1994

He is an Associate Professor with the Department of Mobile Communications, Institute Eurécom, Sophia-Antipolis, France. He was with the European Space Agency (ESTEC), Noordwijk, The Netherlands in 1995. He has been a Visiting Researcher with the Institute Eurécom, Sophia Antipolis, France in 1996 and Princeton University in summer 1997. He has been an Assistant Professor in Telecommunications with the Politecnico di Torino from 1994 to 1998. $\mathrm{He}$ is a co-author of more than 30 papers in international journals and more than 60 in international conferences, and he is the author of three international patents with the European Space Agency. His interests are focused on digital communications theory, information theory, coding theory, and multiuser detection, with particular focus on wireless terrestrial and satellite applications.

Dr. Caire was a recipient of the AEI G. Someda Scholarship in 1991, the COTRAO Scholarship in 1996, and a CNR Scholarship in 1997. He is an Associate Editor for CDMA and Multiuser Detection of the IEEE TRANSACTIONS ON COMMUNICATIONS and Associate Editor for Communication Theory of the Journal of Communications and Network (JCN).

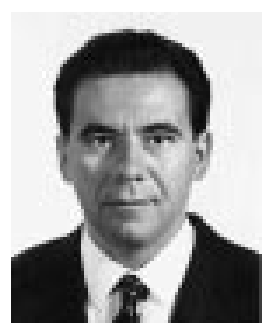

Giorgio Taricco (M'91) was born in Torino (Italy) in 1961. He received the Dr.Engr. degree in electrical engineering from Politecnico di Torino, Italy in 1985.

From 1985 to 1987, he was with CSELT (Italian Telecom Labs), working on the design and definition of the GSM communication system with special regard to the performance of the channel coding subsystem. Since 1991, he has been with the Dipartimento di Elettronica, Politecnico di Torino where he is a Professor of Analog and Digital Communications. In 1996, he was a Research Fellow at ESTEC. His research interests are in the areas of error-control coding, digital communications, multiuser detection, and information theory with applications to mobile communication systems. Currently, he is the co-author of about 40 papers in international journals, 80 papers in international conferences, and two international patents with CSELT. 
Emanuele Viterbo (M'95) was born in Torino, Italy, in 1966. He received the Laurea degree in electrical engineering in 1989 and the Ph.D. degree in 1995 in electrical engineering, both from the Politecnico of Torino, Torino, Italy.

From 1990 to 1992, he was with the European Patent office, The Hague, The Netherlands, as a Patent Examiner in the field of dynamic recording and in particular, in the field of error-control coding. Between 1995 and 1997, he held a postdoctoral position in the Dipartimento di Elettronica, Politecnico di Torino in Communications Techniques over Fading Channels. Between 1997 and 1998 , he was a Visiting Researcher with the Information Sciences Research Center, AT\&T Research, Florham Park, NJ. Since 1998, he has been an Assistant Professor with Dipartimento di Elettronica, Politecnico di Torino. His current research interests are in lattice codes for the Gaussian and fading channels, algebraic coding theory, digital terrestrial television broadcasting, and digital magnetic recording.

Dr. Emanuele Viterbo was awarded a NATO Advanced Fellowship in 1997 from the Italian National Research Council. 